\title{
Spatial conductivity mapping of carbon nanotube composite thin films by electrical impedance tomography for sensing applications
}

\author{
Tsung-Chin Hou ${ }^{1}$, Kenneth $\mathbf{J} \operatorname{Loh}^{1}$ and Jerome P Lynch ${ }^{1,2,3}$ \\ ${ }^{1}$ Department of Civil and Environmental Engineering, University of Michigan, Ann Arbor, \\ MI 48109-2125, USA \\ 2 Department of Electrical Engineering and Computer Science, University of Michigan, \\ Ann Arbor, MI 48109-2125, USA \\ E-mail: jerlynch@umich.edu
}

Received 22 April 2007, in final form 8 June 2007

Published 6 July 2007

Online at stacks.iop.org/Nano/18/315501

\begin{abstract}
This paper describes the application of electrical impedance tomography (EIT) to demonstrate the multifunctionality of carbon nanocomposite thin films under various types of environmental stimuli. Carbon nanotube (CNT) thin films are fabricated by a layer-by-layer $(\mathrm{LbL})$ technique and mounted with electrodes along their boundaries. The response of the thin films to various stimuli is investigated by relying on electric current excitation and corresponding boundary potential measurements. The spatial conductivity variations are reconstructed based on a mathematical model for the EIT technique. Here, the ability of the EIT method to provide two-dimensional mapping of the conductivity of CNT thin films is validated by (1) electrically imaging intentional structural defects in the thin films and (2) mapping the film's response to various $\mathrm{pH}$ environments. The ability to spatially image the conductivity of CNT thin films holds many promises for developing multifunctional CNT-based sensing skins.
\end{abstract}

(Some figures in this article are in colour only in the electronic version)

\section{Introduction}

The emerging field of nanotechnology puts forth nanomaterials with desired mechanical, electrical, and chemical functionality at the macroscopic scale [1]. Advances in the field have aided the development of multifunctional materials that are capable of achieving several functional goals within a single platform, such as displaying mechanical strength coupled with sensing and actuation capabilities [2, 3]. Much interest has surrounded the development of carbon nanotube (CNT) composites which are commonly composed of singlewalled (SWNT) and multi-walled carbon nanotubes (MWNT) embedded within a polymer matrix. CNT composites take

3 Address for correspondence: Department of Civil and Environmental Engineering, 2380 G G Brown Building, Ann Arbor, MI 48109-2125, USA. advantage of the impressive mechanical, electrical and physical properties of individual nanotubes to produce materials endowed with similar bulk properties [4-8]. For example, mechanically strong CNT composites that simultaneously exhibit conductivity changes to external stimuli (i.e. to $\mathrm{pH}$ [6], strain [7, 8], humidity [9] and light [10]) have been reported, thereby allowing the material to be used in a variety of sensing applications.

The development of multifunctional CNT composites for sensing applications hinges on accurate monitoring of the bulk electrical properties of the material before, during and after exposure to external stimuli. Various measurement techniques have been widely used to quantify the resistance of solid state and thin film materials. Most common are direct current (DC) techniques where a constant current, $I$, is injected 
into a sample while the voltage, $V$, across the sample is measured [11]. If the points of current injection and voltage measurement are collocated, the approach is termed the twopoint probe method. If the sample resistance, $R$, is low, contact impedance of the probes can introduce measurement errors. To minimize the influence of contact impedance, the four-point probe method separates the points of current and voltage measurements to acquire a more accurate measurement of sample resistance [12]. Using knowledge of the specimen geometry (e.g. film thickness), bulk material conductivity, $\sigma$, can be calculated using the measured resistance, $R$. To more precisely analyse the electrical properties of materials whose resistance is dependent on the frequency of an applied alternating current (AC), electrical impedance spectroscopy (EIS) can be used in lieu of DC probe methods [13]. In EIS, the amplitude and phase relationship between an applied AC signal and the measured voltage is encapsulated within an impedance measurement, $Z(\omega)$, where $\omega$ is the cyclic frequency of the injected current. Impedance is a complexvalued electrical property of the specimen with the real and imaginary impedance components dependent upon the resistance and capacitance of the specimen, respectively.

An inherent limitation of both DC probe and EIS methods is the assumption of relative homogeneity of the specimen's electrical properties between probe points. Should the electrical properties of a specimen be spatially inhomogeneous, both methods average the spatial variations to provide an equivalent homogeneous resistance or impedance measurement. To offer spatial resolution of specimen resistance, adaptations of the DC probe methods have been offered. Scanning four-point probe methods have been proposed with probe measurements repeatedly made at equally spaced probe locations defined by a grid [14]. This method can be scaled down into a scanning electron microscope (SEM) environment to offer a spatial resolution of a few microns [15]. Other established techniques include scanning spreading resistance microscopy which consists of a conductive atomic force microscope tip that measures spreading resistance profiles across a specimen cross section [16]. While such methods provide a mapping of resistance over multiple dimensions, they require repeated time-consuming measurements that are conducted at the macro- or microscales.

With the emergence of multifunctional thin film composites capable of sensing stimuli such as strain and $\mathrm{pH}$ [6], there is a need for analytical methods that can map the thin film resistance over multiple spatial dimensions. In this paper, a novel approach to mapping the distribution of conductivity of CNT composites based upon electrical probing at the specimen boundary is described. Termed electrical impedance tomography (EIT), the method has been successfully applied in other fields including medical imaging of cancerous growths in animal tissue [17]. The method differs from scanning probe methods since electrical measurements need only be taken at the boundary of a specimen. Based on voltage measurements taken at the film boundary when an AC signal is applied, EIT inversely solves the Laplace equation to reconstruct a twodimensional (2D) map of the specimen conductivity within the measured film boundary. EIT is especially powerful in applications where the CNT composite is implemented as a sensing skin, since mapping of film conductivity provides direct spatial depictions of the external stimulus inducing the conductivity changes. In this paper, the theory of conductivity reconstruction by EIT is presented followed by its implementation on layer-by-layer assembled carbon nanotube composite thin films. The method is validated by intentionally manufacturing films with defects consistent with non-uniform conductivity distributions. Furthermore, the use of nanotube-based composite thin films as a sensing skin is illustrated by mapping changes in film conductivity to different $\mathrm{pH}$ environments using EIT.

\section{Electrical impedance tomography}

\subsection{Mathematical model of the forward problem}

The flow of electrical current within a conductive or semiconductive thin film body, $\Omega$, can be described at any point by the 2D Laplace equation:

$$
\nabla \cdot[\sigma(x, y) \nabla \phi(x, y)]=-I(x, y)=0
$$

where $\sigma$ is the film conductivity, $\phi$ is the electrical potential and $I$ is the current. The two in-plane dimensions of the thin film are designated by the position variables $x$ and $y$. In equation (1), the gradient of electric potential $(\nabla \phi)$ is equivalent to the electric field vector, E. For ohmic materials, the current density vector, $\mathbf{J}$, at a point is related to $\mathbf{E}$ by Ohm's law $(\mathbf{J}=\sigma \mathbf{E})$; hence, the divergence of the current density is equal to current $(\nabla \cdot \mathbf{J}=I)$. If electrical current is only applied to the film boundary and no current is applied to or generated from the film interior, then the Laplace equation is written by $I(x, y)=0$.

Should an alternating current be applied in lieu of direct current, then equation (1) can be modified to reflect the frequency of the AC signal, $\omega$ :

$$
\begin{gathered}
\nabla \cdot[\gamma(x, y, \omega) \nabla \phi(x, y, \omega)]=0 \\
\gamma=\sigma+\mathrm{i} \omega \varepsilon
\end{gathered}
$$

with conductivity replaced by complex admittivity, $\gamma$, which is dependent on conductivity, frequency and permittivity, $\varepsilon$. The Laplace equation offers a unique solution for electric potential, $\phi(x, y)$, if the voltage along the body boundary is measurable (Dirichlet boundary condition) and the line integral of current along the boundary is zero (Neumann boundary condition) [17]:

$$
\oint I(\omega) \partial \Omega=0
$$

where $\partial \Omega$ is the boundary of $\Omega$. The Laplace equation (equation (2)) with Dirichlet and Neumann boundary conditions applied is referred to as the continuum model of the forward problem [18]. If considering only the real part of admittivity, Kohn and Vogelius [19] have shown that this model is still valid with a unique and strictly positive conductivity function, $\sigma(x, y)$. In the forward problem, the electric potential can be found based upon a known distribution of admittance and applied current. Equation (3) requires a continuous analytical function describing current along the boundary; measurement of boundary current along the entirety of the boundary is not practical. Based on this fact and 
(a)
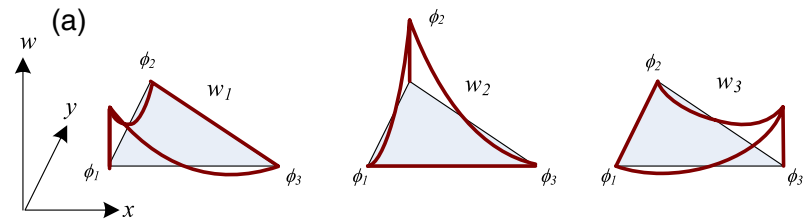

(b)

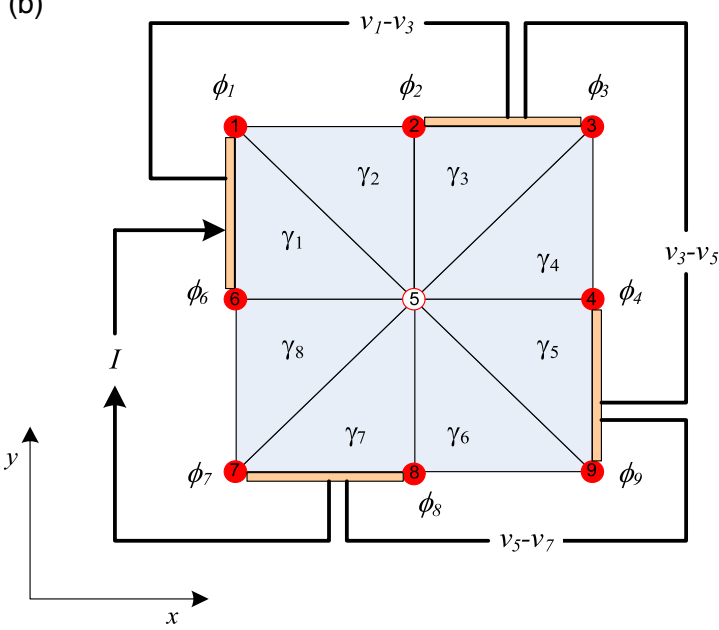

Figure 1. Weak FEM formulation of the Laplace equation: (a) second-order polynomial basis functions, $w_{i}$, corresponding to a triangular element; (b) eight element body with boundary current, I, and voltages, $v$.

other practical issues, the complete electrode model has been proposed by Somersalo et al [20]. This model describes the boundary in a discrete manner and considers the effects of shunting and contact impedance at the electrodes, thereby providing a more realistic mathematical model for practical implementation.

\subsection{Finite element formulation of the forward problem}

Solving equation (2) for an analytical solution is generally not feasible; as a result, the finite element method (FEM) is often used to approximate a solution [21]. The FEM solution begins by using discrete elements to represent the body $(\Omega)$. In the FEM approach, each element is assumed to have constant material properties (e.g. conductivity). The variation in electric potential along the boundary of each element $\left(\Omega_{\mathrm{E}}\right)$ is assumed to be a linear sum of potential at the $i$ th element vertex multiplied by second-order parabolic basis functions, $w_{i}$. Assuming triangular elements, the potential along the element boundary is defined by the three vertices (figure 1(a)):

$$
\left.\phi\right|_{\Omega_{\mathrm{E}}}=\sum_{i=1}^{3} \phi_{i} w_{i}
$$

Equation (4) will not satisfy the continuous form of the Laplace equation (equation (2)). Hence, variational methods are used to derive the weak form of the Laplace equation [22]. The result is a linear set of equations for each triangular element of the body that relates the electric potential at each element vertex $\left(\phi_{i}\right)$, the measured voltage on the boundary of the element $\left(v_{n}\right)$ and applied current $(I)$. A global set of linear equations can be formed by assembling the equations of each element in the body mesh to form:

$$
\left[\begin{array}{cc}
A_{M}+A_{z} & A_{W} \\
A_{W}^{\mathrm{T}} & A_{D}
\end{array}\right]\left\{\begin{array}{l}
\Phi \\
V
\end{array}\right\}=\left\{\begin{array}{l}
0 \\
I
\end{array}\right\}
$$

where the system matrix, $\mathbf{A}$, is a square matrix that is calculated based upon the linear basis functions, $w$, and the assumed element conductivities, $\sigma$. For a given applied current to the body boundary, the electric potential at each mesh node and voltage on the film boundary can be determined. For example, the thin film body in figure 1(b) is meshed using eight elements defined by nine nodes. If a current, $I$, is sourced at element 1 and drained at element 7, then equation (5) can be solved for the electric potential at the nine nodes $(\Phi=$ $\left.\left\{\begin{array}{llll}\phi_{1} & \phi_{2} & \ldots & \phi_{9}\end{array}\right\}^{\mathrm{T}}\right)$ and for the voltage measured at the three adjacent electrodes $\left(V=\left\{\begin{array}{llll}v_{1} & v_{3} & v_{5} & v_{7}\end{array}\right\}^{\mathrm{T}}\right)$. It should be noted that, in this example, $\mathbf{A}$ is a $13 \times 13$ square matrix. Provided the system matrix is often sparse, LU decomposition is generally required to solve the forward problem [17].

\subsection{The inverse problem}

Electrical impedance tomography (EIT) is the inverse of the forward problem where the interior distribution of conductivity (real part of admittivity) is calculated from boundary voltage measurements corresponding to known currents applied at the body boundary. The general approach to solving the EIT inverse problem is to find the conductivity of each element, such that the forward problem provides a prediction of the boundary voltages asymptotically close to those experimentally measured. The inverse problem seeks to find the conductivity distribution that minimizes the quadratic cost function, $f$ :

$$
f(\sigma)=\frac{1}{2}\|\phi(\sigma)-v\|^{2} .
$$

The general approach is to repeatedly solve the forward problem as the conductivity distribution is varied between successive forward problems. To minimize the quadratic cost function, various algorithms are available that offer an optimal means of adjusting the conductivity distribution between successive forward solutions; in this study, the regularized Gauss-Newton algorithm, which is an iterative approach, is adopted to minimize equation (6) [18]. The inverse solution to the Laplace equation represents an ill-posed inverse problem defined by an underdetermined set of linear equations. As a result, a single set of boundary voltage measurements to one application of current is insufficient to accurately map the film conductivity. Rather, EIT requires redundancy in the experimental data with repeated sets of boundary voltage measurements taken while current is separately applied at multiple boundary elements.

\section{Experimental details}

\subsection{Materials}

Purified single-walled carbon nanotubes (SWNT) with ash content less than $13 \mathrm{wt} \%$ are obtained from Carbon Nanotechnologies, Inc. Thin film polyelectrolyte constituents, namely poly(aniline) emeraldine base (PANI, $M_{\mathrm{w}} \approx$ 100000 ), poly(vinyl alcohol) (PVA) and poly(sodium styrene4-sulfonate) (PSS, $\left.M_{\mathrm{w}} \approx 1000000\right)$, are purchased from 
Table 1. Summary of LbL SWNT-composite thin films and their corresponding LbL constituents fabricated for EIT spatial conductivity mapping.

\begin{tabular}{llll}
\hline Polycationic specie & Polyanionic specie & $\begin{array}{l}\text { Number of } \\
\text { bilayers }\end{array}$ & $\begin{array}{l}\text { Thin film } \\
\text { characteristics }\end{array}$ \\
\hline $1.0 \mathrm{wt} \%$ PANI in 1.0 vol\% DMF & $\begin{array}{l}0.8 \mathrm{mg} \mathrm{ml}^{-1} \\
\text { SWNT in } 1.0 \mathrm{wt} \%\end{array}$ & 50 and 100 & $\begin{array}{l}\text { Low conductivity } \\
\text { and pH-sensitive }\end{array}$ \\
& $\begin{array}{l}\text { PSS } \\
0.8 \mathrm{mg} \mathrm{ml}^{-1}\end{array}$ & 50 and 100 & High conductivity \\
& SWNT in $1.0 \mathrm{wt} \%$ & & \\
\hline & PSS & & \\
\hline
\end{tabular}

Sigma-Aldrich. The remaining reagents were obtained from Fisher-Scientific. Electrical contacts between the thin film and electrodes are established using colloidal silver paste and copper tape, both of which are purchased from Ted Pella.

\subsection{Nanocomposite fabrication methodology}

The fabrication of homogeneous nanostructured multifunctional thin films is attained via a layer-by-layer (LbL) selfassembly method [23]. Through alternate dipping of a charged substrate (e.g. glass or silicon) in oppositely charged polyelectrolyte and suspended nanomaterial species, a thin film of controlled morphology with no phase segregation can be deposited one monolayer at a time. The alternate nanometre-thick monolayers of polyelectrolyte and nanomaterials are held by weak interaction forces such as electrostatic and van der Waals forces. Through judicious selection of polyelectrolytic species used during LbL self-assembly, multifunctional nanocomposites have been successfully demonstrated [6].

In this study, LbL assembly begins by dipping a charged glass substrate treated with piranha solution $(3: 7$ by vol $\mathrm{H}_{2} \mathrm{O}_{2}: \mathrm{H}_{2} \mathrm{SO}_{4}$ ) in a polycationic (1.0 wt $\%$ PANI dissolved in $1.0 \mathrm{vol} \% \mathrm{DMF}$ or $1.0 \mathrm{wt} \%$ PVA in deionized water) solution for $5 \mathrm{~min}$. Upon rinsing with $18 \mathrm{M} \Omega$ deionized water for 3 min followed by drying with compressed nitrogen for $10 \mathrm{~min}$, the substrate, along with its adsorbed monolayer, is then immersed in a polyanionic solution. Enhancement of thin film conductivity and $\mathrm{pH}$ sensitivity is accomplished via the use of a steric stabilized SWNT in $1.0 \mathrm{wt} \%$ PSS solution to yield a stable polyanionic suspension after ultrasonication bath $(90 \mathrm{~min})$ and probe sonication treatment (90 min) (as outlined in [6]). The LbL process continues by immersing the substrate in the prepared SWNT-PSS solution for $5 \mathrm{~min}$, followed by rinsing and drying for 3 and 10 min, respectively. The aforementioned procedure yields one bilayer of the thin film and is denoted as $(A / B)_{n}$, where $A$ and $B$ represent the oppositely charged species and $n$ represents the number of bilayers. Realization of electrically conductive and mechanically strong sensing skins for EIT spatial conductivity mapping is accomplished by fabricating (SWNT-PSS/PANI $)_{50},(\text { SWNT-PSS/PANI })_{100}$ and (SWNT-PSS/PVA) $)_{50}$ thin films (table 1). From the SEM image of figure 2, it can be seen that only individual and small bundles of SWNTs are deposited in the polymer matrix composite during LbL self-assembly.

\subsection{Nanocomposite thickness measurement}

In order to ensure accurate EIT calculations of thin film conductivity, the precise thickness of each specimen is

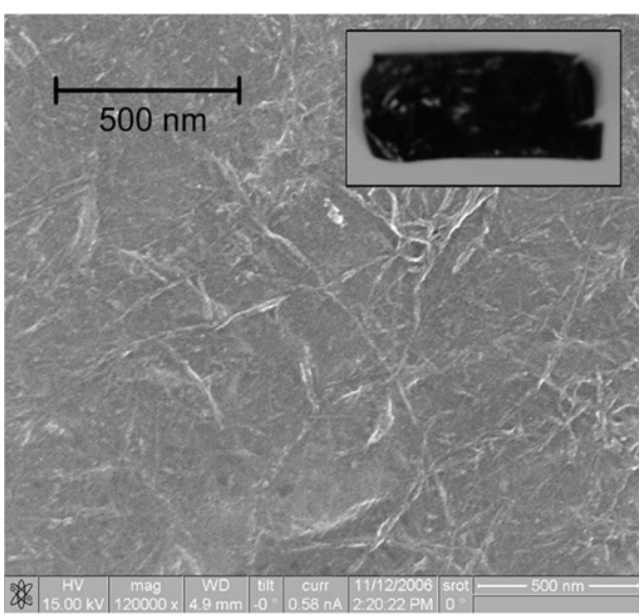

Figure 2. An SEM image of a (SWNT-PSS/PVA) $)_{50}$ LbL thin film shows the selective deposition of individual and small bundles of nanotubes within the polymer matrix. Rinsing during layer-by-layer self-assembly removes excessively large agglomerated particles. The inset depicts the corresponding free-standing (SWNT-PSS/PVA) $)_{50}$ thin film upon lift-off from its original glass substrate.

measured using a J A Woollam spectroscopic ellipsometer. The entire set-up of the spectroscopic ellipsometer consists of a Base-160 sample holder with a QTH-200 light source connected to an EC-270 electronic control module and data acquisition system (DAQ). Since ellipsometry thickness measurements require thin films deposited on a reflective surface, LbL SWNT composites are fabricated onto a silicon substrate (with a $38 \mathrm{~nm}$ thick oxide layer, University Wafers). By taking 16 total ellipsometry thickness measurements at 16 unique locations throughout the thin film surface, the average film thickness and thickness gradient is determined.

\subsection{Boundary electrical measurement}

Spatial conductivity mapping of CNT composite thin films by EIT is achieved by measuring current-voltage relationships across a thin film's boundary. In this study, electrical potential measurement of thin films is performed using electrical contacts made by drying colloidal silver paste $(\sim 1 \mathrm{~mm}$ wide) equidistantly spaced $(\sim 2 \mathrm{~mm})$ between strip copper header pins (DigiKey) and the film surface. For instance, SWNT multilayer thin film specimens roughly $25 \times 25 \mathrm{~mm}^{2}$ in area are instrumented with eight electrodes along each of the four sides of the film boundary (totaling 32 electrodes). In this study, electrode placement is precise because of the use of 

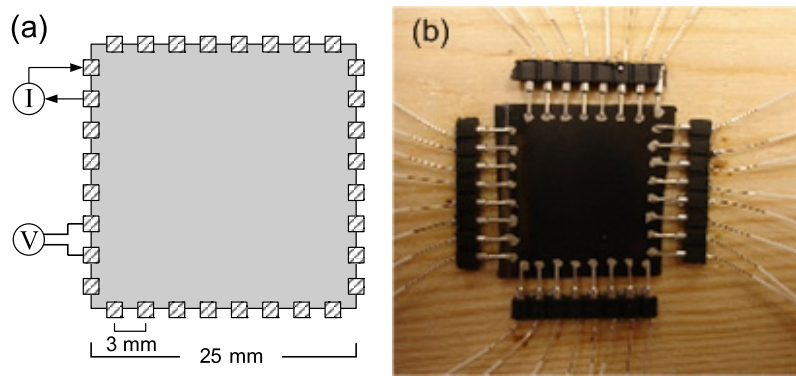

Figure 3. (a) Illustration of boundary electrode placement for conductivity mapping; (b) LbL thin film specimen with boundary electrodes installed. The specimen is mounted to a wood stand to prevent detachment of copper wire from the thin film electrodes.

header pins manufactured with a pin-to-pin spacing of $2 \mathrm{~mm}$. However, should other electrode placement methods be used that lead to inaccurate placement, the electrode positions would be inconsistent with those specified in the EIT forward problem and minor distortions of the final conductivity map could occur. Upon drying (after $6 \mathrm{~h}$ ), the aforementioned silver electrodes are connected to a National Instruments (NI) DAQ. Minimization of experimental error is prevented by housing specimens in a customized wood platform to prevent undesirable detachments between electrodes and copper wires. Figure 3(a) gives a schematic view of electrode placement while figure 3(b) shows a thin film specimen resting on the wood platform with 32 electrodes installed along its boundaries.

While many methods have been proposed to measure the actual boundary potential of a solid body [17], an adjacent electrode measurement technique is employed herein. By injecting a regulated alternating current using a current source (Keithley 6221) at a pair of adjacent electrodes, the NIDAQ with a rack-mounted multiplexed terminal block is employed to simultaneously measure the potential between the remaining pairs of electrodes. Upon determining the electrode boundary potentials, the current source and its corresponding current sink are shifted to the next adjacent electrode pair. The aforementioned process is cycled until all electrode pairs have been subjected to $\mathrm{AC}$ excitation. If the boundary has $L$ electrodes, then the total number of independent boundary potential measurements made in the adjacent electrode technique is $L(L-1) / 2$. Higher resolution (i.e. finer mesh) of conductivity mapping can be reached by increasing the number of electrodes, $L$, to obtain a larger set of independent boundary measurements.

\subsection{Two-dimensional $p H$ sensing}

Based on a previous study conducted by Loh et al [6], electrochemical $\mathrm{pH}$ sensitivity can be encoded within thin films using SWNT and a poly(aniline) emeraldine base solution. Different levels of charge injection from pipetted $\mathrm{pH}$ buffer solutions ( $\mathrm{pH} 1-10)$ lead to dramatic conductivity variations at areas where the $\mathrm{pH}$ buffer solutions come into direct contact with the (SWNT-PSS/PANI ${ }_{n}$ thin film surface [6]. For $\mathrm{pH}$ studies, the fabricated (SWNT-PSS/PANI) ${ }_{n}$ thin films (roughly $25 \times 60 \mathrm{~mm}^{2}$ in area) are modified by mounting five plastic wells to its surface using high-vacuum grease (Dow Corning).
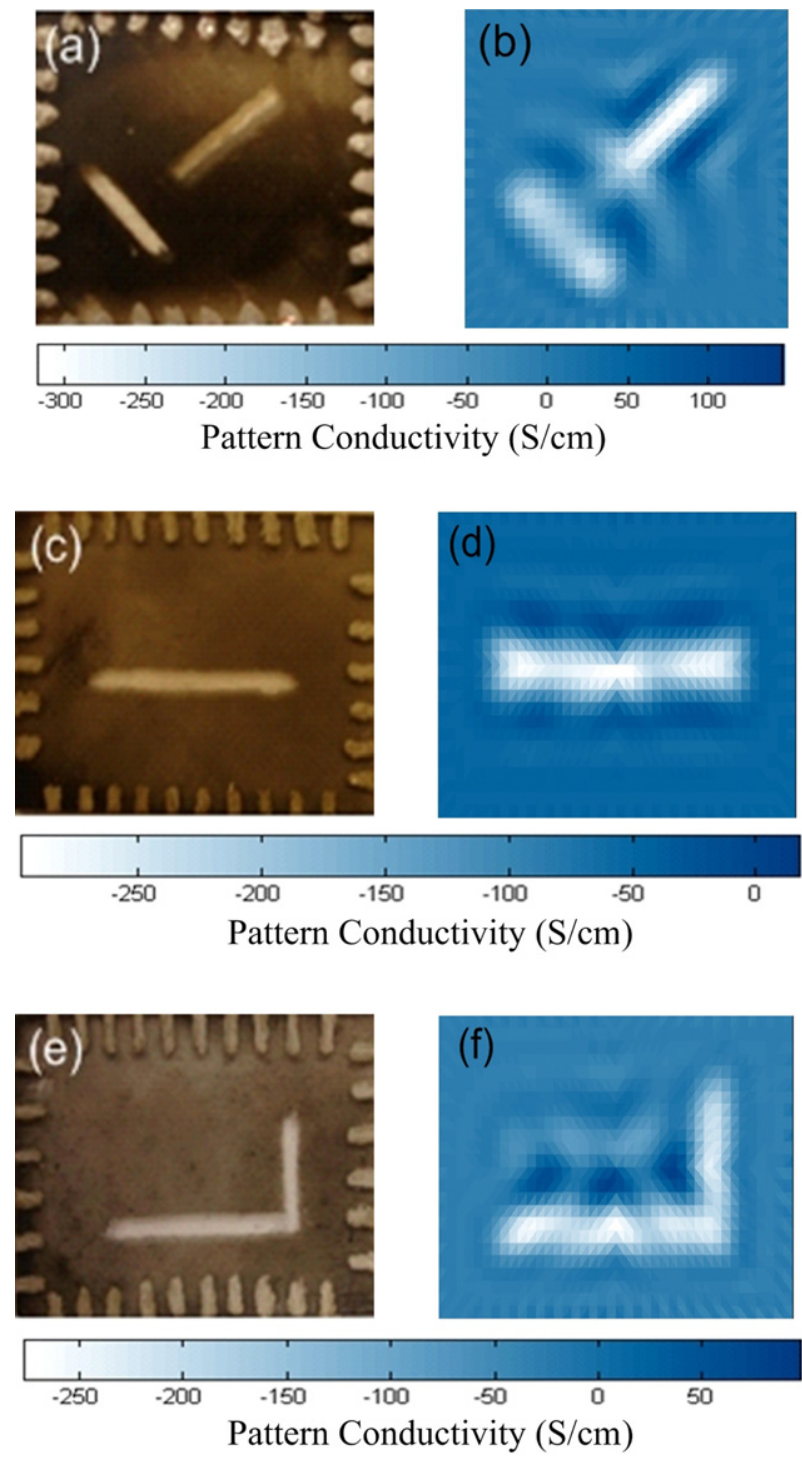

Figure 4. Pattern conductivity maps $\left(\sigma_{\text {pattern }}\right)$ of three types of physically etched thin film specimens. (a), (c) and (e) are optical pictures of thin films with double slashes, straight line and L shaped etchings; (b), (d) and (f) are the corresponding pattern conductivity maps of (a), (c) and (e) with the colour scale in units of S/cm.

Each plastic well serves as a chamber for containment of the $\mathrm{pH}$ buffer solutions while boundary potentials are measured for 2D EIT conductivity mapping of the specimen, thereby allowing simultaneous sensing of different $\mathrm{pH}$ solutions on a single thin film specimen. In this EIT experimental set-up, a total of 48 electrodes are applied.

\section{Results and discussion}

\subsection{EIT validation of conductivity inhomogeneity}

Validation of EIT conductivity mapping of LbL thin films is conducted by assessing conductivity homogeneity variations among four separate (SWNT-PSS/PVA) ${ }_{50}$ thin film specimens. Following LbL fabrication, EIT is performed to obtain baseline conductivity maps $\left(\sigma_{\text {base }}\right)$ for each thin film specimen. After the 
(a)
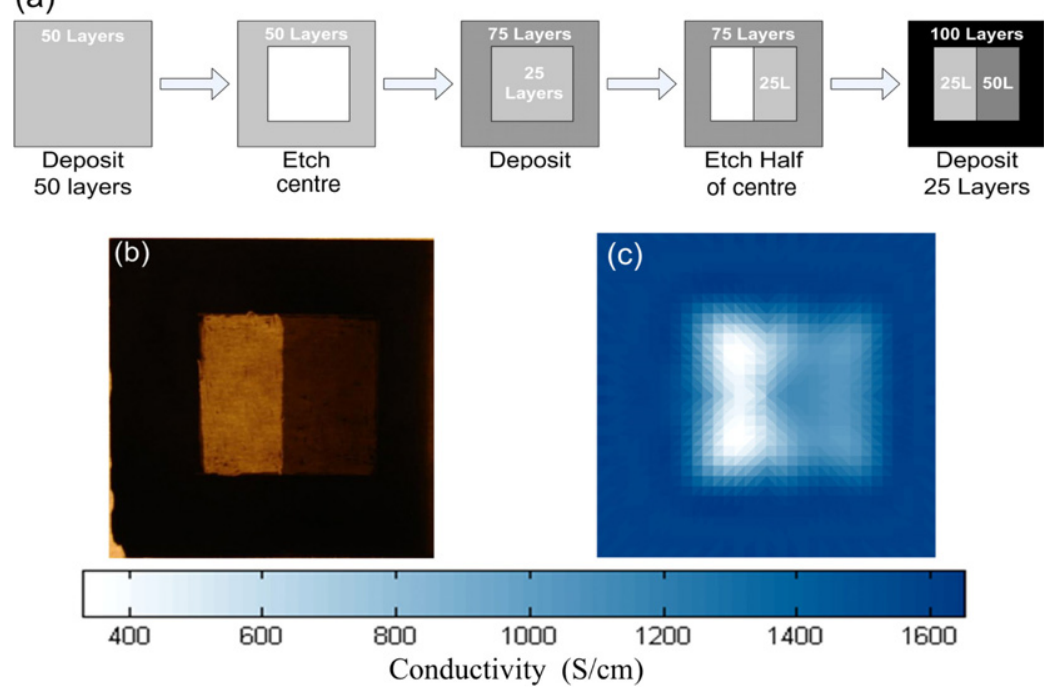

Figure 5. (a) Sequential deposition and etching process to structure three regions of different conductivity in a single thin film; (b) back-lighted image of thin film specimen with 3 dipping layers, 25 (left centre), 50 (right centre) and 100 layers (outer field); (c) is the conductivity map of (b).

baseline conductivity maps are reconstructed, an intentional inhomogeneity is introduced within the film structure by physically etching straight, diagonal and L-shaped cuts into the film surface (figures 4(a), (c) and (e)) to create regions of zero conductivity. After etching, EIT is performed again on each thin film specimen to obtain the etched conductivity maps $\left(\sigma_{\text {etch }}\right)$. These maps are then subtracted from $\sigma_{\text {base }}$ to obtain associated pattern conductivity maps, $\sigma_{\text {pattern. }}$ From figures $4($ b), (d) and (f), it can be observed that EIT can clearly identify the change in conductivity within the thin film structure. When compared with the back-lighted photographs of the three aforementioned thin film specimens, the experimental EIT conductivity maps (figures 4(b), (d) and (f)) correspond precisely with the photographs (figures 4(a), (c) and (e)).

In order to demonstrate that the EIT technique can identify subtle changes in conductivity within the thin film structure, three levels of conductivity are encoded into a fourth specimen by controlling the number of bilayers deposited. From a previous study conducted by Loh et al [24], thin film conductivity increases in tandem with increasing number of bilayers deposited. Thus, fabrication of this fourth specimen with varied conductivity is accomplished by initially fabricating a (SWNT-PSS/PVA $)_{50}$ thin film. Upon mechanical etching of an $18 \times 18 \mathrm{~mm}^{2}$ window in the middle of the film to form a region of zero conductivity, the LbL process continues to fabricate another (SWNT-PSS/PVA) ${ }_{25}$ thin film structure over the etched 50-bilayer film. Again, mechanical etching is employed to remove a $9 \times 18 \mathrm{~mm}^{2}$ window before another 25-bilayer thin film is deposited (figure 5(a)); the final thin film structure consists of three different magnitudes of conductivity. Generally, darker regions of the backlighted image (figure 5(b)) suggest greater carbon nanotube deposition and hence increasing film conductivity. Using only a 32-electrode boundary set-up, EIT successfully identifies major and minor conductivity variations due to different CNT deposition densities (figure 5(c)).

\subsection{EIT validation of measured absolute conductivity}

As presented in section 2, the EIT method can reconstruct an absolute measure of thin film conductivity simply through boundary potential measurements. While it has been demonstrated that relative conductivity changes can be accurately identified via EIT (section 4.1), no experimental data suggest the calculated conductivity map corresponds to the true conductivity of the thin film. Here, a validation case study is presented to directly compare EIT conductivity estimates to experimental data obtained using traditional DC two-point probing [12]

Similar to section 4.1, the validation of absolute thin film spatial conductivity is conducted using $25 \times$ $25 \mathrm{~mm}^{2}$ (SWNT-PSS/PVA) ${ }_{50}$ thin films fabricated on a silicon substrate (figure 6(a)). The pristine film is subjected to EIT to obtain its corresponding spatial conductivity map (where a total of 32 electrodes are used as described in section 3.4). Upon boundary potential measurements and EIT reconstruction, the thin film is physically sliced to form a $4 \times 4$ element grid with each element electrically isolated from all others (figure 6(b)). By drying colloidal silver paste between each grid element, individual element resistance $(R)$ is measured via an Agilent 64401A digital multimeter connected in a two-point probe fashion. Once resistance is obtained for a grid element, the multimeter electrodes are removed and applied to the corresponding electrodes of the next element. This is repeated until all 16 mesh resistances have been determined. Since film resistivity, $\rho$ (or equivalently conductivity, $\sigma$ ) is dependent on thin film thickness $(h)$, the thickness of each thin film element is measured using ellipsometry (as detailed in section 3.3). Resistivity, $\rho$, is calculated by:

$$
\rho=\frac{1}{\sigma}=\frac{R}{l} w h
$$

where $w$ is the element's width and $l$ is the distance between the two-point probe electrodes. 

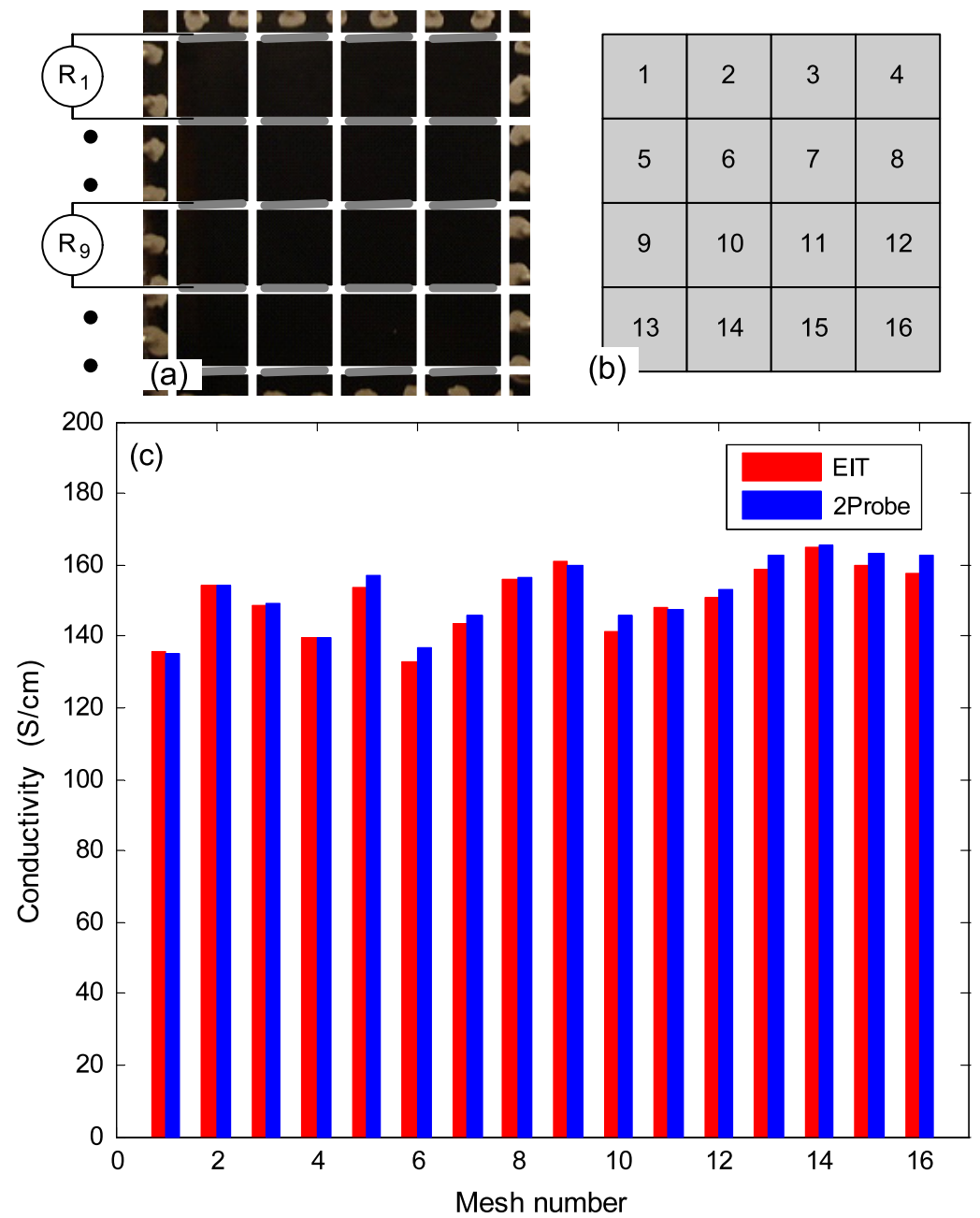

Figure 6. Thin film conductivity validation: (a) thin film specimen is equally meshed into 16 individual elements by physical slicing of the thin film; (b) the mesh number of (a); (c) the conductivity comparison of (a) between EIT and the two-probe method (average error is $2.1 \%$ ).

The EIT reconstructed conductivity map using 32 boundary electrodes is compared to the conductivity measured for each of the mesh elements by two-point probing. To make a direct comparison, the triangular elements of the EIT conductivity map that fall within the domain of each grid element are averaged. As presented in figure 6(c), the average conductivity measured by EIT reconstruction is within $2.1 \%$ error of those measured by using two-point probe methods for each grid element. These results suggest the conductivity maps offered by EIT reconstruction are accurate as compared with more traditional conductivity measurement methods.

\section{3. $p H$ sensing skin}

In previous work, (SWNT-PSS/PANI) ${ }_{n}$ thin films have been shown to drastically increase in film resistance in tandem with increasing $\mathrm{pH}$ buffer solutions $(\mathrm{pH} 1-10)$ [6]. With a $\mathrm{pH}$ sensitivity of approximately $20.66 \mathrm{k} \Omega \mathrm{cm}^{-2} / \mathrm{pH}$, these thin films serve as ideal candidates for $\mathrm{pH}$ sensing in a wide variety of applications (e.g. corrosion monitoring). The electrochemical response of (SWNT-PSS/PANI) $n$ thin films have been characterized with entire films exposed to a single $\mathrm{pH}$ environment while two-point probe measurements are conducted [6]. In contrast, EIT conductivity mapping offers a direct method of sensing $\mathrm{pH}$ variations across the entire thin film.

Demonstration of the potential functionality of (SWNT$\mathrm{PSS} / \mathrm{PANI})_{n}$ thin films for distributed $\mathrm{pH}$ sensing is conducted using the experimental set-up described in section 3.5 (figure 7(a)). Prior to the application of $\mathrm{pH}$ buffer solutions in the five wells, the conductivity map of the thin film is reconstructed by EIT. This map will serve as a baseline map to which further conductivity maps will be compared. To illustrate the use of (SWNT-PSS/PANI) ${ }_{n}$ thin films for biological applications, a small range of $\mathrm{pH}$ buffer solutions ranging from 5 to 9 are pipetted into the five wells. The EITderived conductivity maps of the thin film exposed to $\mathrm{pH}$ buffer solutions are subtracted from the baseline conductivity, thereby revealing changes in conductivity due to $\mathrm{pH}$. First, the surface of the thin film is exposed to $\mathrm{pH}$ buffer solutions corresponding to $\mathrm{pH}$ values of $7,7.5,8,8.5$ and 9 ; figure 7 (b) reveals the conductivity of the (SWNT-PSS/PANI) 100 thin film decreases in linear proportion to $\mathrm{pH}$. Second, the basic solutions are removed and acid buffer solutions with $\mathrm{pH}$ values 7, 6.5, 6, 5.5 and 5 are applied to the film surface. Figure 7(c) confirms the thin film undergoes an increase in the film conductivity in 

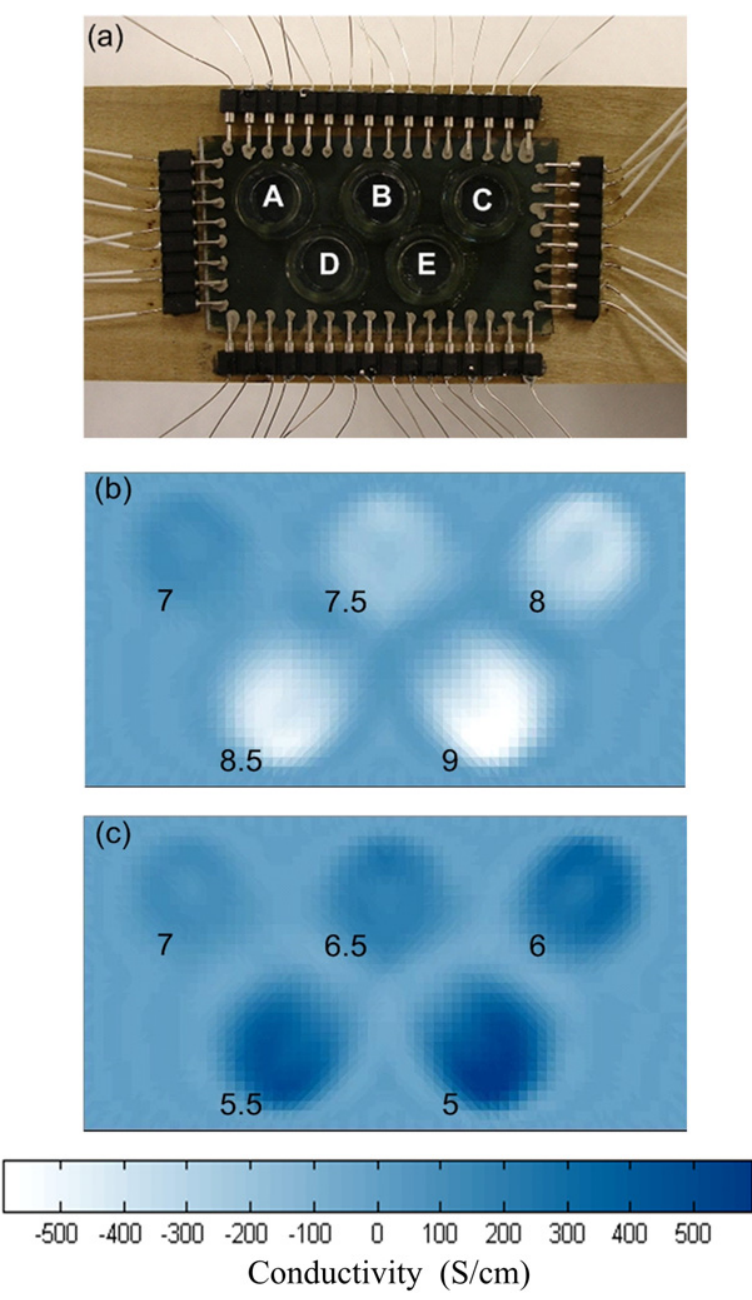

Figure 7. (a) (SWNT-PSS/PANI) $)_{100}$ thin film under several magnitudes of $\mathrm{pH}$ stimuli; (b) change in spatial conductivity when $\mathrm{pH}$ in well $\mathrm{A}, \mathrm{B}, \mathrm{C}, \mathrm{D}$ and $\mathrm{E}$ is $7,7.5,8,8.5$ and 9 , respectively; (c) change in spatial conductivity when $\mathrm{pH}$ in well $\mathrm{A}, \mathrm{B}, \mathrm{C}, \mathrm{D}$ and $\mathrm{E}$ is $7,6.5,6,5.5$ and 5 , respectively.

tandem with acidic $\mathrm{pH}$. Figure 8 summarizes the sensitivity of (SWNT-PSS/PANI) ${ }_{n}$ thin film composites to $\mathrm{pH}$ stimuli including earlier work conducted by the same group [25]. As shown, a linear relation exists in the $\mathrm{pH}$ sensitivity of electrochemically active (SWNT-PSS/PANI) ${ }_{n}$ thin films.

\section{Conclusions}

In this study, electrical impedance tomography is employed to map the two-dimensional spatial conductivity distribution among layer-by-layer assembled carbon nanotube-polyelectrolyte composite thin films. First, validation of conductivity inhomogeneity is performed on four (SWNT-PSS/PVA) 100 thin film specimens. By mechanically etching different patterns within three of the films and fabricating three regions of different conductivity in the fourth, EIT spatial conductivity can accurately identify regions of inhomogeneous conductivity. Furthermore, to ensure that the mapped conductivity corresponds to the true conductivity of the thin film specimen, EIT results are compared with those

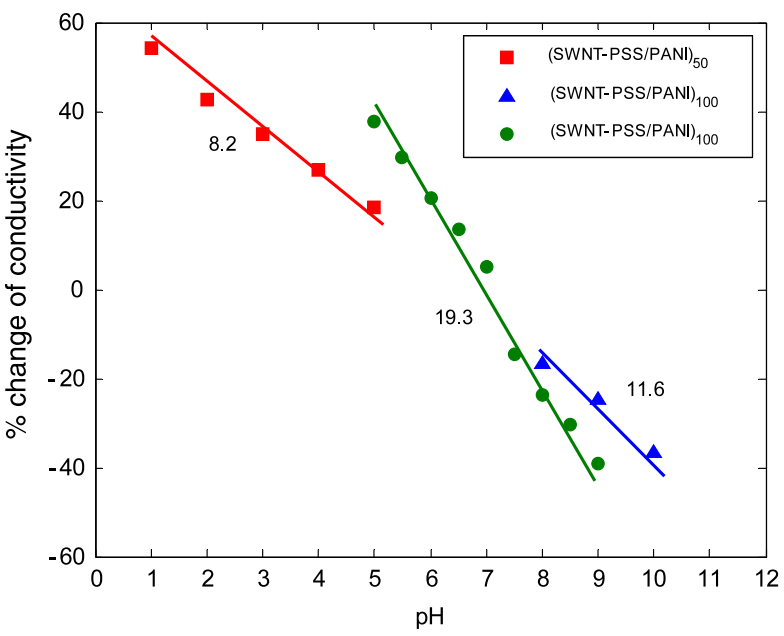

Figure 8. Sensitivity of three different (SWNT-PSS/PANI) ${ }_{n}$ thin films under $\mathrm{pH}$ stimuli. All three films exhibit similar linear variations in conductivity as a result of $\mathrm{pH}$ buffer solutions applied to the film surface.

measured using a DC two-point probe technique. When comparing EIT measured conductivity to measurements taken by two-point probe techniques, strong agreement (within 2\%) is encountered.

With the accuracy of the EIT conductivity reconstruction established, the study focuses upon array sensing of $\mathrm{pH}$ using the surface of a single (SWNT-PSS/PANI) $n$ thin film specimen. The conductivity maps of the film exposed to different $\mathrm{pH}$ buffer solutions in five locations reveal the outline of the solution wells. Furthermore, the change in conductivity of the film surface immediately below each circular well is shown to be linearly proportional to $\mathrm{pH}$. The EIT method is clearly a powerful approach for measuring the conductivity of CNT thin film composites. The approach is elegant since repeated probe measurements need not be taken to measure thin film conductivity. As a result, multifunctional CNT composite films could be used in a variety of sensing applications where spatial depiction of conductivity changes to stimuli is desired (e.g. corrosion monitoring of metallic surfaces). While multilayered carbon nanotubepolyelectrolyte composite films are used as case studies in this work, it should be noted that the EIT approach to distributed sensing is rather general and can be applied to map the conductivity of other thin film materials. For example, the EIT technique can be easily applied to spatially image $\mathrm{pH}$-induced conductivity changes of other polypyrrole and polyaniline composite films $[26,27]$.

\section{Acknowledgments}

This research is supported by the National Science Foundation under program manager Dr S C Liu (grant no. CMS-0528867). The authors would like to express their gratitude to Dr Nadine Wong Shi Kam for suggesting the use of EIT mapping of $\mathrm{pH}$-sensitive CNT thin films. Furthermore, the authors would also like to thank the University of Michigan Electron Microbeam Analysis Laboratory (UM-EMAL) for providing support with SEM imaging as well as Professor Joerg Lahann 
and Mr Hsien-Yeh Chen for providing assistance with thin film ellipsometry thickness measurements. Last, but not least, the authors would like to express their gratitude towards Professor Nicholas Kotov for providing valuable assistance and feedback throughout this research study.

\section{References}

[1] Cao G 2004 Nanostructures and Nanomaterials (London: Imperial College Press)

[2] Thomas J P and Qidwai M A 2004 Mechanical design and performance of composite multifunctional materials Acta Mater. 52 2155-64

[3] Schulz M J, Kelkar A D and Sundaresan M J 2006 Nanoengineering of Structural, Functional, and Smart Materials (Boca Raton, FL: CRC Press)

[4] Skakalova V, Dettlaff-Weglikowska U and Roth S 2005 Electrical and mechanical properties of nanocomposites of single wall carbon nanotubes with PMMA Synth. Mater. 152 349-52

[5] Thostenson E T and Chou T-W 2006 Carbon nanotube networks: sensing of distributed strain and damage for life prediction and self healing Adv. Mater. 18 2837-41

[6] Loh K J, Kim J, Lynch J P, Kam N W S and Kotov N A 2007 Multifunctional layer-by-layer carbon nanotube-polyelectrolyte thin films for strain and corrosion sensing Smart Mater. Struct. 16 429-38

[7] Kang I, Schulz M J, Kim J H, Shanov V and Shi D 2006 A carbon nanotube strain sensor for structural health monitoring Smart Mater. Struct. 15 737-48

[8] Zhang W, Suhr J and Koratkar N 2006 Carbon nanotube/polycarbonate composites as multifunctional strain sensors J. Nanosci. Nanotechnol. 6 960-4

[9] Yu H, Cao T, Zhou L, Gu E, Yu D and Jiang D 2006 Layer-by-layer assembly and humidity sensitive behavior of poly(ethyleneimine)/multiwall carbon nanotube composite films Sensors Actuators B 119 512-5

[10] Valentini L, Mengoni F, Armentano I, Kenny J M, Ricco L, Alongi J, Trentini M, Russo S and Mariani A 2006 Enhancement of photoelectrical properties in polymer nanocomposites containing modified single-walled carbon nanotubes by conducting dendrimer J. Appl. Phys. 99114305

[11] Mayer J W and Lau S S 1990 Electronic Materials Science: for Integrated Circuits in Si and GaAs (New York, NY: Macmillan Publishing)

[12] Smits F M 1958 Measurement of sheet resistivities with the four-point probe Bell Syst. Tech. J. 37 711-8
[13] Barsoukov E and Macdonald J R 2005 Impedance Spectroscopy: Theory, Experiment, and Applications (Hoboken, NJ: Wiley)

[14] Hansen T M, Stokbro K, Hansen O, Hassenkam T, Shiraki I, Hasegawa S and Boggild P 2003 Resolution enhancement of scanning four-point-probe measurements on two-dimensional systems Rev. Sci. Instrum. 74 3701-8

[15] Hasegawa S, Shiraki I, Tanikawa T, Petersen C L, Hansen T M, Boggild P and Grey F 2002 Direct measurement of surface-state conductance by microscopic four-point probe method J. Phys.: Condens. Matter 14 8379-92

[16] Xu M W, Hantschel T and Vandervorst W 2002 Three-dimensional carrier profiling of InP-based devices using scanning spreading resistance microscopy Appl. Phys. Lett. 81 177-9

[17] Holder D S 2005 Electrical Impedance Tomography-Methods, History, and Applications (Bristol: Institute of Physics Publishing)

[18] Borcea L 2002 Electrical impedance tomography Inverse Problems 18 R99-136

[19] Kohn R V and Vogelius M 1984 Determining conductivity by boundary measurement Commun. Pure Appl. Math. 37 113-23

[20] Somersalo E, Cheney M and Isaacson D 1992 Existence and uniqueness for electrode model for electric current computed tomography SIAM J. Appl. Math. 52 1023-40

[21] Hughes T J R 2000 The Finite Element Method: Linear Static and Dynamic Finite Element Analysis (Mineola, NY: Dover)

[22] Vauhkonen M, Vauhkonen P J, Kaipio J P and Karjalainen P A 1997 Three-dimensional electrical impedance tomography using complete electrode model Proc. SPIE 3171 166-74

[23] Decher G and Schlenoff J B 2003 Multilayer Thin Films. Sequential Assembly of Nanocomposite Materials (Federal Republic of Germany: Wiley-VCH)

[24] Loh K J, Lynch J P, Shim B S and Kotov N A 2007 Tailoring piezoresistive sensitivity of multilayer carbon nanotube composite sensors J. Intell. Mater. Syst. Struct. at press

[25] Hou T, Loh K J and Lynch J P 2007 Electrical impedance tomography of carbon nanotube composite materials Proc. SPIE 652926-1-10

[26] Ferrer-Anglada N, Kaempgen M and Roth S 2006 Transparent and flexible carbin nanotube/polupyrrole and carbon nanotube/polyaniline $\mathrm{pH}$ sensors Phys. Status Solidi 243 3519-23

[27] Aquino-Binag C N, Kumar N, Lamb R N and Pigram P J 1996 Fabrication and characterization of a hydroquinonefunctionalized polypyrrole thin-film $\mathrm{pH}$ sensor Chem. Mater 82579 